\title{
Circulation Marine De La Baie De Dakhla (Sud Du Maroc) Par Modèle Hydrodynamique 2d
}

\author{
Hilmi Karim, PhD \\ Orbi Abdellatif, PhD \\ Makaoui Ahmed, PhD \\ Institut National de Recherche Halieutique, Maroc \\ Bouksim Hassan, PhD \\ Direction de la Météorologie Nationale, Maroc \\ Idrissi Mohammed, PhD \\ Ettahiri Omar, PhD \\ Abdellaoui Benyounes, PhD \\ Ait Chattou Mustapha, PhD \\ Institut National de Recherche Halieutique, Maroc
}

\begin{abstract}
The marine circulation of Dakhla bay (South of Morocco $23^{\circ} 30^{\prime} \mathrm{N}$ $16^{\circ} \mathrm{W}$ ) have been studied from January to February 2010 by $2 \mathrm{D}$ hydrodynamic model. This study aims to understand the marine circulation of this bay and its hydrodynamic functioning. Mainly governed by M2 tide and during spring tides, tidal currents are very strong in intensity near the inlet of the bay and its surrounding (between 1 and $2 \mathrm{~m} \cdot \mathrm{s}^{-1}$ ). In its central part and the nothern part of the bay, their intensity are very less $\left(<1 \mathrm{~m} \cdot \mathrm{s}^{-1}\right)$. During neap tides, tidal currents are less in intensity $\left(<0.6 \mathrm{~m} \cdot \mathrm{s}^{-1}\right)$ in all the areas of the bay.
\end{abstract}

Keywords: Dakhla - bay - South of Morocco- marine circulation- 2D hydrodynamic model

\section{Résumé}

La circulation marine de la baie de Dakhla (Sud du Maroc 233’N$\left.16^{\circ} \mathrm{O}\right)$ a été appréhendée sur le mois de janvier à février 2010 par modélisation hydrodynamique bidimensionnelle (2D). Cette étude vise à mieux comprendre la circulation marine de la baie et son mode de fonctionnement hydrodynamique. Les marées, principalement la marée semi diurne de type M2, dominent les courants de marée au sein de la baie. 
Durant les marées de vives eaux, les courants sont très intenses, en particulier à l'entrée de la baie et aux zones avoisinantes (entre 1 et $2 \mathrm{~m} . \mathrm{s}^{-1}$ ). Dans sa partie centrale et en allant vers la partie Nord de la baie, ces courants diminuent d'intensité $\left(<1 \mathrm{~m} \cdot \mathrm{s}^{-1}\right)$. Durant les marées de mortes eaux, ces courants de marée présentent généralement des intensités généralement inférieures à $0.6 \mathrm{~m} . \mathrm{s}^{-1}$ dans toutes les zones de la baie.

Mots clés: Dakhla- baie - Sud du Maroc - Circulation marine- Modèle hydrodynamique $2 \mathrm{D}$

\section{Introduction}

\section{Présentation du site d'étude}

La baie de Dakhla (ou baie de «Oued Eddahab»), située le long du littoral saharien marocain $\left(23^{\circ} 30^{\prime} \mathrm{N}-16^{\circ} \mathrm{W}\right)$, est parmi les plus importantes baies situées au sud du Maroc (Figure 1a). Elle est séparée de l'océan Atlantique par la péninsule de Oued Eddahab et présente une longueur de 37 $\mathrm{km}$ environ avec une largeur variant entre 10 à $12 \mathrm{~km}$ environ. Orientée dans l'axe NNE-SSW, elle communique avec l'Océan Atlantique du côté Sud à travers une large passe de $13 \mathrm{~km}$ environ d'ouverture (Figure $1 \mathrm{~b}$ ).

\section{Climat de la zone d'étude}

\section{Température de l'air}

Observées sur la période 1986-2015, les températures moyennes mensuelles de l'air à la station de Dakhla varient entre $11.7^{\circ} \mathrm{C}$ et $28.8^{\circ} \mathrm{C}$; la valeur minimale étant observée au mois de février 2012 et la valeur maximale au mois d'octobre 1995 avec une période «chaude» allant généralement d'août à octobre/novembre et une période «fraîche» allant de décembre à février (Figure 2). Les températures moyennes minimales de l'air varient entre $11.7^{\circ} \mathrm{C}$ et $21.3^{\circ} \mathrm{C}$ et les températures moyennes maximales entre $20.3^{\circ} \mathrm{C}$ et $28.8^{\circ} \mathrm{C}$ sur la période $1986-2015$ (Figure 2).
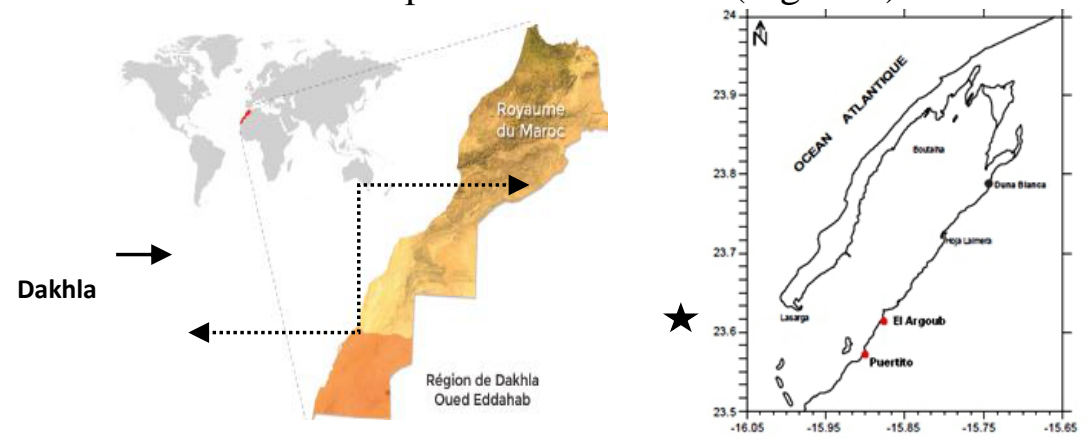

Figure 1. Situation géographique de la région de Dakhla Oued Eddahab (à gauche 1.a) et de la baie de Dakhla (à droite 1.b) (adapté de ANDA, 2015 et Zidane et al., 2008). 
Ainsi, les écarts thermiques sont faibles ne dépassant pas $10^{\circ} \mathrm{C}$ au maximum entre les saisons à cause de la situation géographique de Dakhla à proximité de l'océan Atlantique et de l'influence océanique très marquée (Source MarocMétéo, 2015).

\section{Précipitation}

Comme pour l'ensemble des régions sahariennes marocaines, le régime pluviométrique de la région de la baie de «Oued Eddahab» est caractérisé, par une grande irrégularité, en plus de son extrême aridité (Agence du Sud, 2014). En termes de normales mensuelles des précipitations, ces dernières varient entre $0.9 \mathrm{~mm}$ à $3.7 \mathrm{~mm}$ pour les mois de janvier à mai, inférieures à $0.2 \mathrm{~mm}$ entre juin et juillet, 2.2 à $6.7 \mathrm{~mm}$ d'août à décembre (Figure 3), ce qui dénote que les précipitations sont généralement concentrées sur deux périodes de l'année: août à octobre et décembre à mars (Source MarocMétéo, 2015).

\section{Moyennes Mensuelles des températures Tx et Tn Station de Dakhla (1986-2015)}

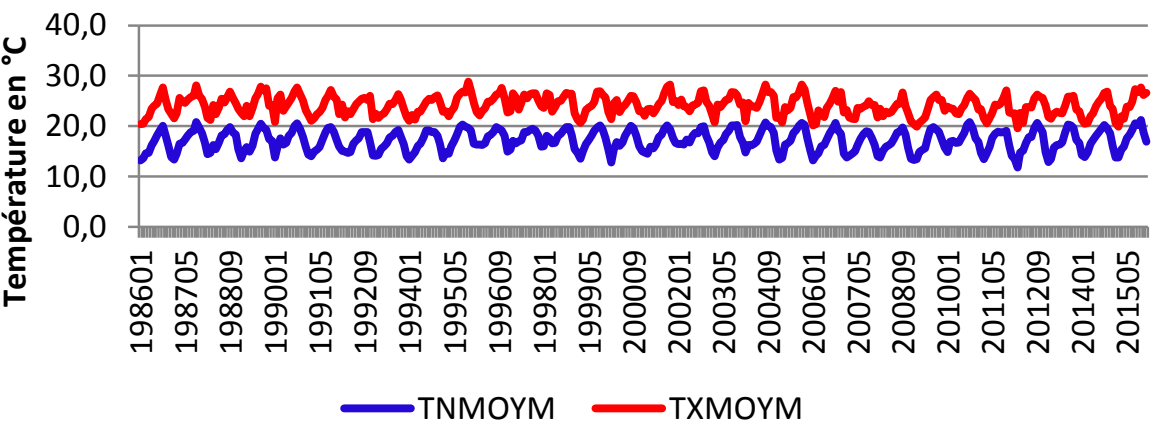

Figure 2. Températures moyennes mensuelles de l'air à Dakhla sur la période 1986-2015. Les températures moyennes minimales mensuelles sont représentées en bleu et les températures moyennes maximales mensuelles en rouge (Source MarocMétéo, 2015). 


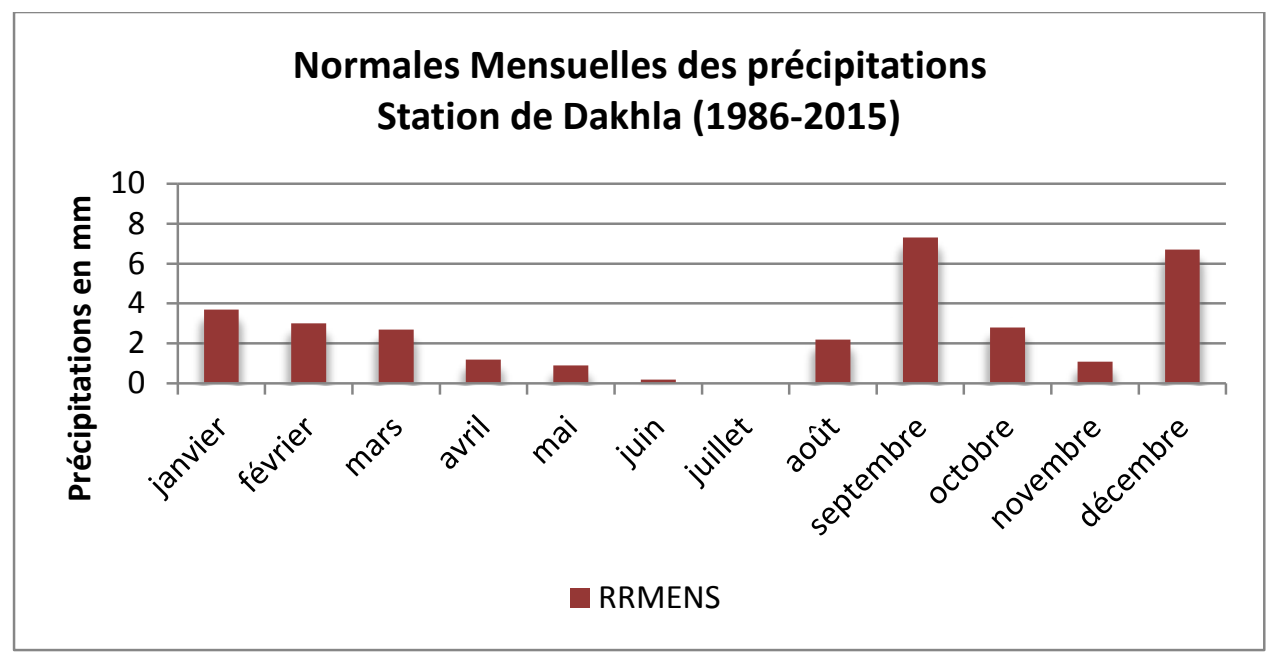

Figure 3. Normales mensuelles des précipitations à la station de Dakhla sur la période 1986-2015 (Source MarocMétéo-2015).

\section{Vents}

La figure 4 illustre les roses mensuelles de vent à la station de Dakhla sur la période 2005-2014 (Source MarocMétéo, 2015). L'analyse de ces roses de vent montre que les vents dominants soufflent généralement du secteur Nord Nord Ouest à Nord Est, avec une très forte prédominance des vents de secteurs Nord (Figure 4) qui sont caractéristiques des vents Alizés dans la zone sud du Maroc. Quelque soient les saisons, les intensités moyennes maximales des vents de secteur Nord sont souvent supérieures à 9 $\mathrm{m} . \mathrm{s}^{-1}$ et ils présentent des fréquences d'occurrence variables selon les saisons, variant entre $12 \%$ et $28 \%$ pour les saisons d'hiver, entre $18 \%$ et $52 \%$ pour les saisons du printemps, entre $16 \%$ et $64 \%$ pour les saisons d'été et entre $22 \%$ et $56 \%$ pour les saisons d'automne (Figure 4 ).

\section{Objectifs de l'étude}

De par sa grande importance socio économique dans la région, la baie de Dakhla a fait l'objet de plusieurs études en aquaculture, visant notamment à définir son organisation hydrologique, écologique, sédimentologique, en aquaculture (ANDA, 2015, Arid et al. , 2005; INRH, 2002; Lakhdar et al., 2010; NOVEC, 2012 a et b; Orbi et al., 1995, 1996; Orbi et Berraho, 1999; Saad et al., 2013, 2015; Zidane, 2011; Zidane et al., 2008, 2013 a et b). Cependant, des études visant la description des processus physiques régissant la baie de Dakhla et son fonctionnement sont très peu nombreuses (Orbi et al., 1995, 1996) et quasi inexistantes sur la modélisation hydrodynamique. L'objectif de ce travail est de décrire, à l'aide d'un modèle hydrodynamique 2D, la circulation marine bidimensionnelle de la baie et de comprendre les 
processus physiques régissant le fonctionnement hydrodynamique de cette baie.

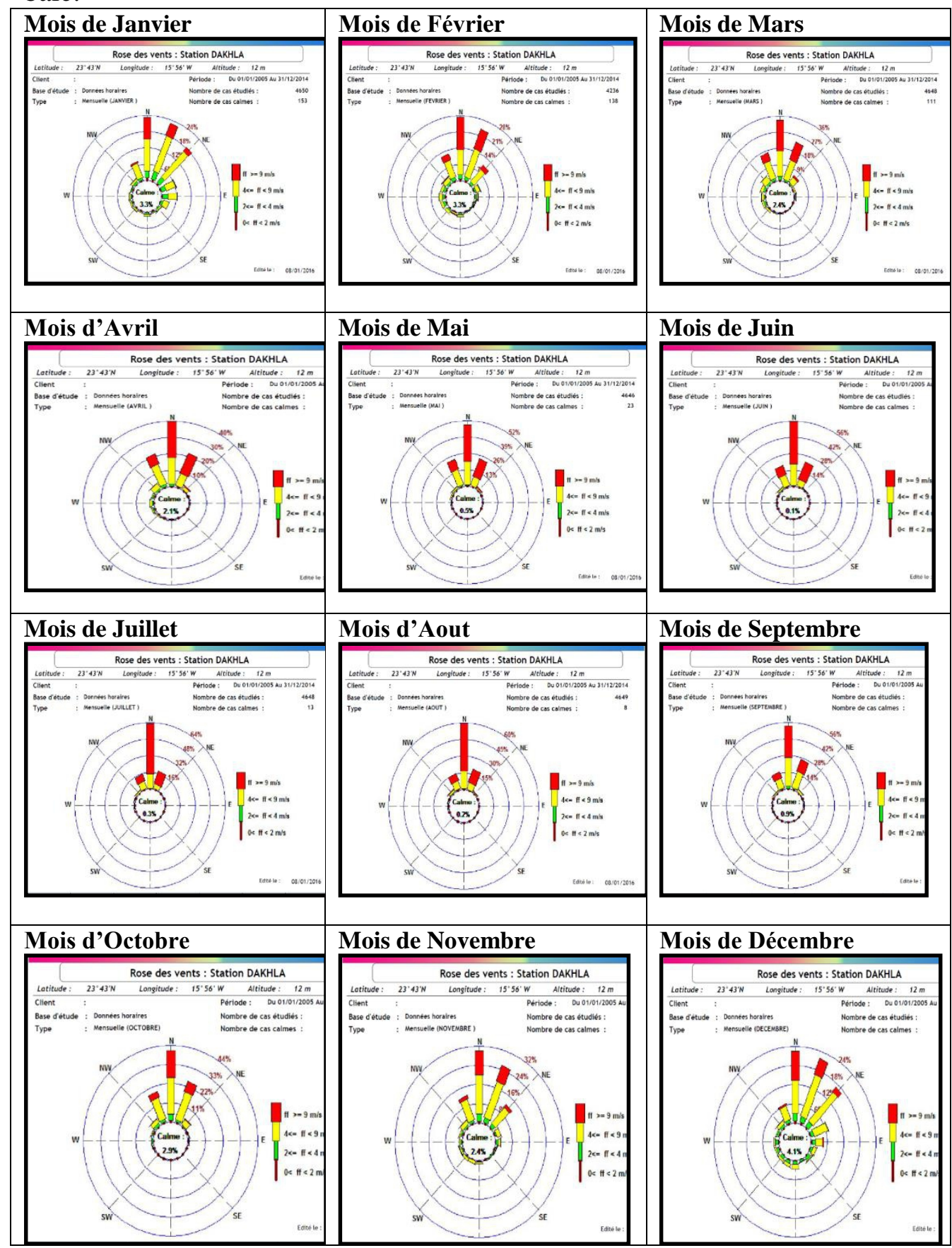

Figure 4 : Rose mensuelles des vents à la station météorologique de Dakhla sur la période 2005-2014 (Source MarocMétéo, 2015). 


\section{Matériel et méthodes}

Le modèle bidimensionnel (2D), implémenté pour la baie de Dakhla, est le modèle MIKE2-HD développé par la société DHI dont les équations du modèle 2D sont décrites en détail (DHI, 1990). Le modèle MIKE21/HD simule l'évolution temporelle des courants et niveau d'eau en intégrant les équations de la conservation du mouvement et de la masse intégrées sur l'axe vertical. Il a été calibré à partir des mesures de courant dans la baie en 2001 (frottement au fond, entrainement par le vent, ...) puis validé par des mesures de courant à la station de la bouée «LA SARGA» située à la position $(23.624147 \mathrm{~N} ;-15.984625 \mathrm{~W})$ (voir position à la figure $1 \mathrm{~b}$ ), sur la période allant du 28 janvier au 09 février 2010 (Nombre Observations horaires $=407$; Moyenne $=-0.01 ; \mathrm{RMS}=0.11 \mathrm{~cm} / \mathrm{s}$; Scatter Index $=-11.45$, CorrCoeff $=0.94$ ). Dans cette version du modèle, ce dernier reproduit parfaitement la marée et à environ 95\% les intensités la composante NordSud du courant qui est la composante dominante du courant dans la baie. Les intrants du modèle 2D sont les suivants:

\section{Bathymétrie de la baie de Dakhla}

La figure 5 présente la bathymétrie de la baie de Dakhla réalisée par l'INRH sur le terrain en 2010 (Lakhdar et al., 2010). Pour les besoins de cette étude en matière de modélisation, la grille de calcul, développée pour le domaine de la baie, comprend 160 mailles en X (vers l'Est) et 180 mailles en Y (vers le Nord), de dimensions $200 \mathrm{~m}$ x $200 \mathrm{~m}$ sur le plan horizontal (Figure 5)

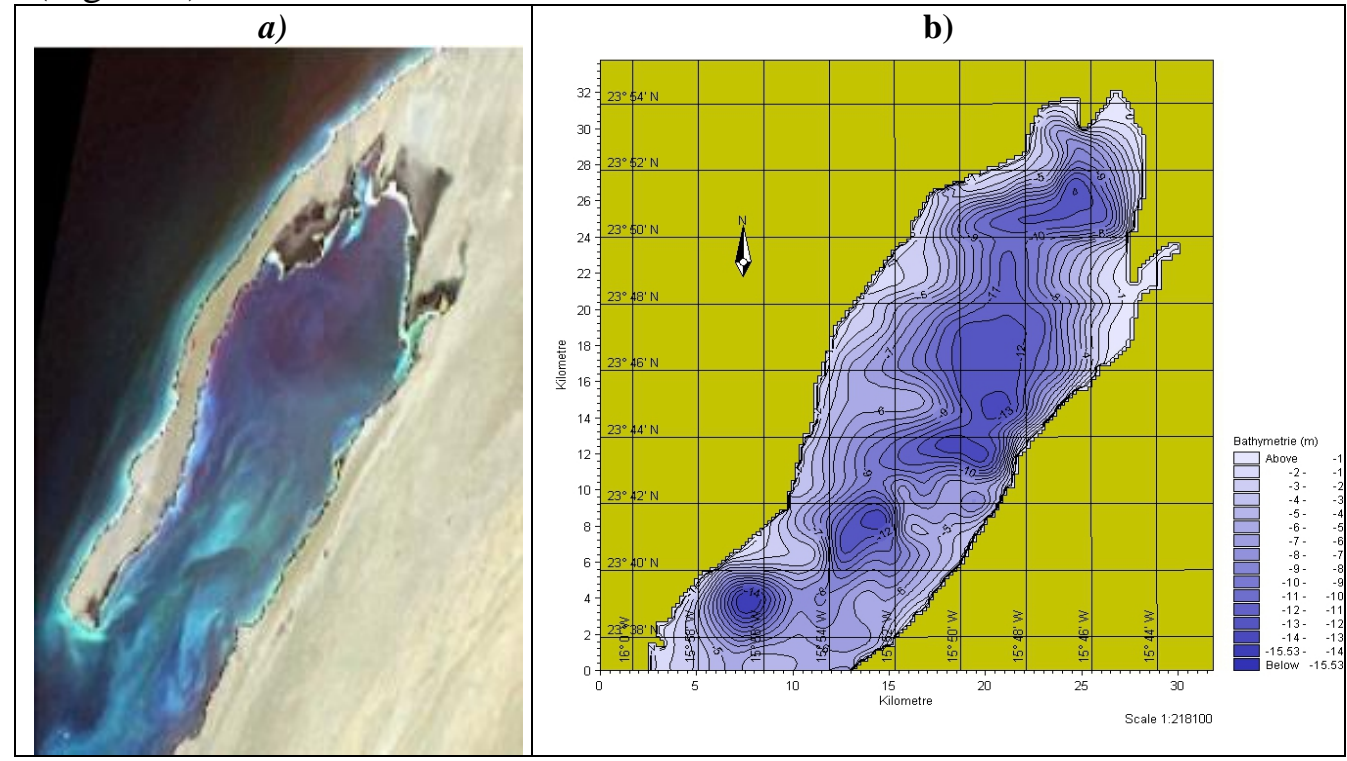

Figure 5: a) image SPOT de la baie de Dakhla en 1993 (Source CRTS) et b) bathymétrie de la baie de Dakha corrigée par rapport à la marée établie par l'INRH en 2010 (d'après Lakhdar et al., 2010). 


\section{Marée}

Pour les besoins du modèle et à ses frontières ouvertes (figure 5 de droite), le modèle 2D a été forcé par la marée observée à l'entrée de la baie au niveau de la bouée de «La Sarga» sus mentionnée sur la période du 28 janvier au 09 février 2010 (Figure 6). Cette période couvre ainsi un cycle de marée compris entre des marées de morte-eau et de vive-eau. Sur cette période de l'année, les coefficients de marée sont compris entre 19 pour les marées de morte eau et 107 pour les marées de vive eau. Ces coefficients sont calculés pour une pleine mer (Source SHOM). C'est le quotient du marnage semi-diurne de la formule harmonique par la valeur moyenne du marnage pour les marées de vive-eau d'équinoxe, admise à 6,1m à Brest (Source Shom). C'est un nombre sans dimension compris entre 20 et 120 et qui varie peu d'un jour sur l'autre. Par convention, le coefficient 100 est attribué au marnage semi-diurne moyen lors des vives-eaux voisines des équinoxes (21 mars, 21 septembre Source SHOM):

- $\quad$ marées extraordinaires de vive-eau d'équinoxe: le marnage à Brest est admis à 7,32 m d'où le coefficient maximal de 120;

- $\quad$ marées de vive-eau moyenne : 95

- $\quad$ marées moyennes : 7

- $\quad$ marées de morte-eau moyenne : 45

- $\quad$ marées de morte-eau les plus faibles ; le marnage à Brest est admis à $1,22 \mathrm{~m}$, d'où le coefficient minimal de 20 .

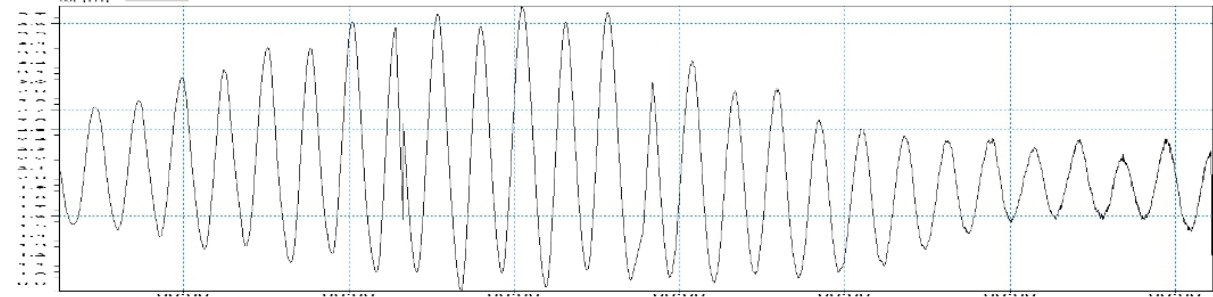

Figure 6. Marée horaire (en $\mathrm{m}$ ) observée au niveau de la bouée de la «Sarga» dans la baie de Dakhla du 28 janvier au 09 février 2010 (Source INRH, 2010).

\section{Vent}

La figure 7 présente la rose du vent tri-horaire sur la période de l'étude s'étalant du 28 janvier au 09 février 2010 (Source données Maroc Météo, 2010). On constate que les vents sont de secteurs variables sur cette période de l'étude, avec des vents dominants (fréquence $>50 \%$ ) dont les intensités sont supérieures à $8 \mathrm{~m} . \mathrm{s}^{-1}$ soufflant du secteur Nord à Nord Est. Ce sont ces vents qui sont généralement observés dans la baie de Dakhla en cette période de l'année (figure 4). Les vents de secteurs Nord Ouest, Est, Sud à Sud Est sont aussi observés mais avec une fréquence moins importante et leurs intensités sont relativement faibles (inférieures à $8 \mathrm{~m}$. s ${ }^{-1}$ ) (Figure 7). 


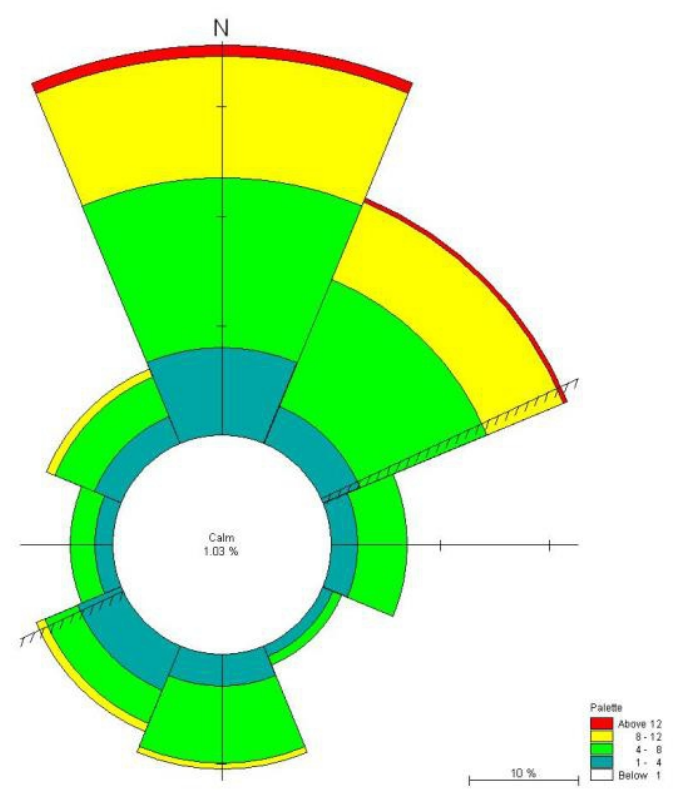

Figure 7. Rose du vent tri horaire observé à la station météorologique de Dakhla sur la période du 01 janvier au 15 février 2010 (Source données Maroc Météo, 2010). L'axe de la baie est représenté sur cette figure en traits hachurés.

\section{Résultats}

Comme mentionné précédemment, la simulation de la circulation marine de la baie de Dakhla a porté sur la période du 28 janvier au 09 février 2010. La baie étant soumise à la forte influence des marées semi diurnes, nous présentons ici quatre exemples de configuration de la circulation de la baie en périodes de marées variables:

$>$ les marées de vive-eau à marée entrante (Figure 8) et à marée sortante (Figure 9). Le coefficient de marée varie entre 103 et 107;

$>$ les marées de morte-eau à marée entrante (Figure 10) et à marée sortante (Figure 11). Le coefficient de marée varie entre 20 et 23. 


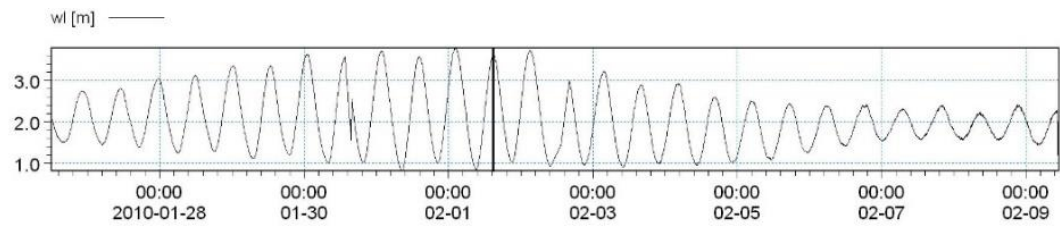

wind speed $[\mathrm{m} / \mathrm{s}]$

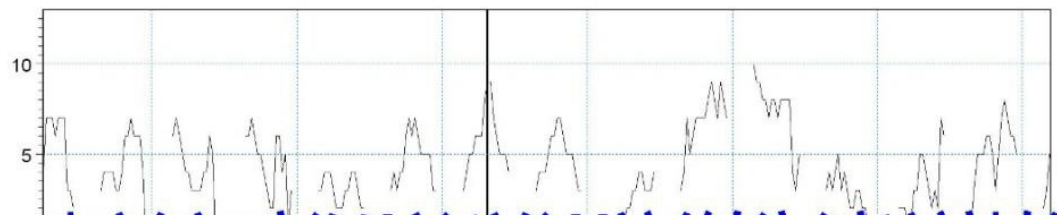

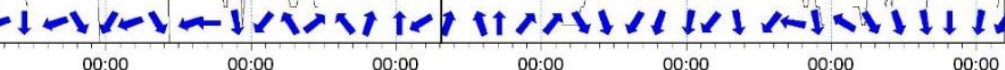
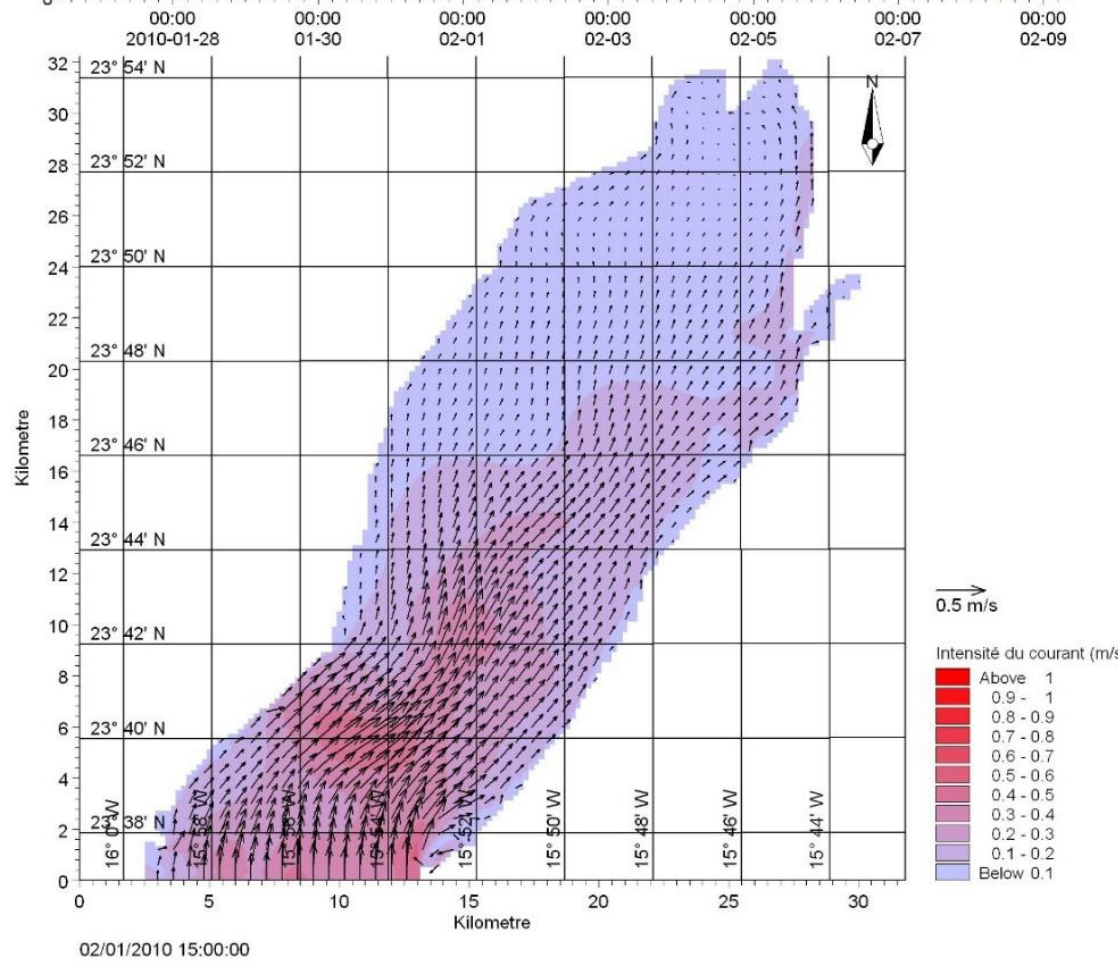

Figure 8. Exemple de circulation de la baie de Dakhla en période de vive-eau à marée entrante (cas du 01 février 2010 à $15 \mathrm{~h}$ - coefficient de marée 103). 


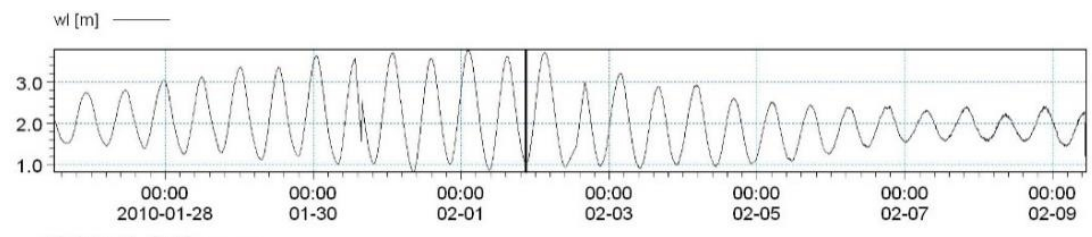

wind speed $[\mathrm{m} / \mathrm{s}]$
wind directon $[\mathrm{deg}]$$\rightarrow$
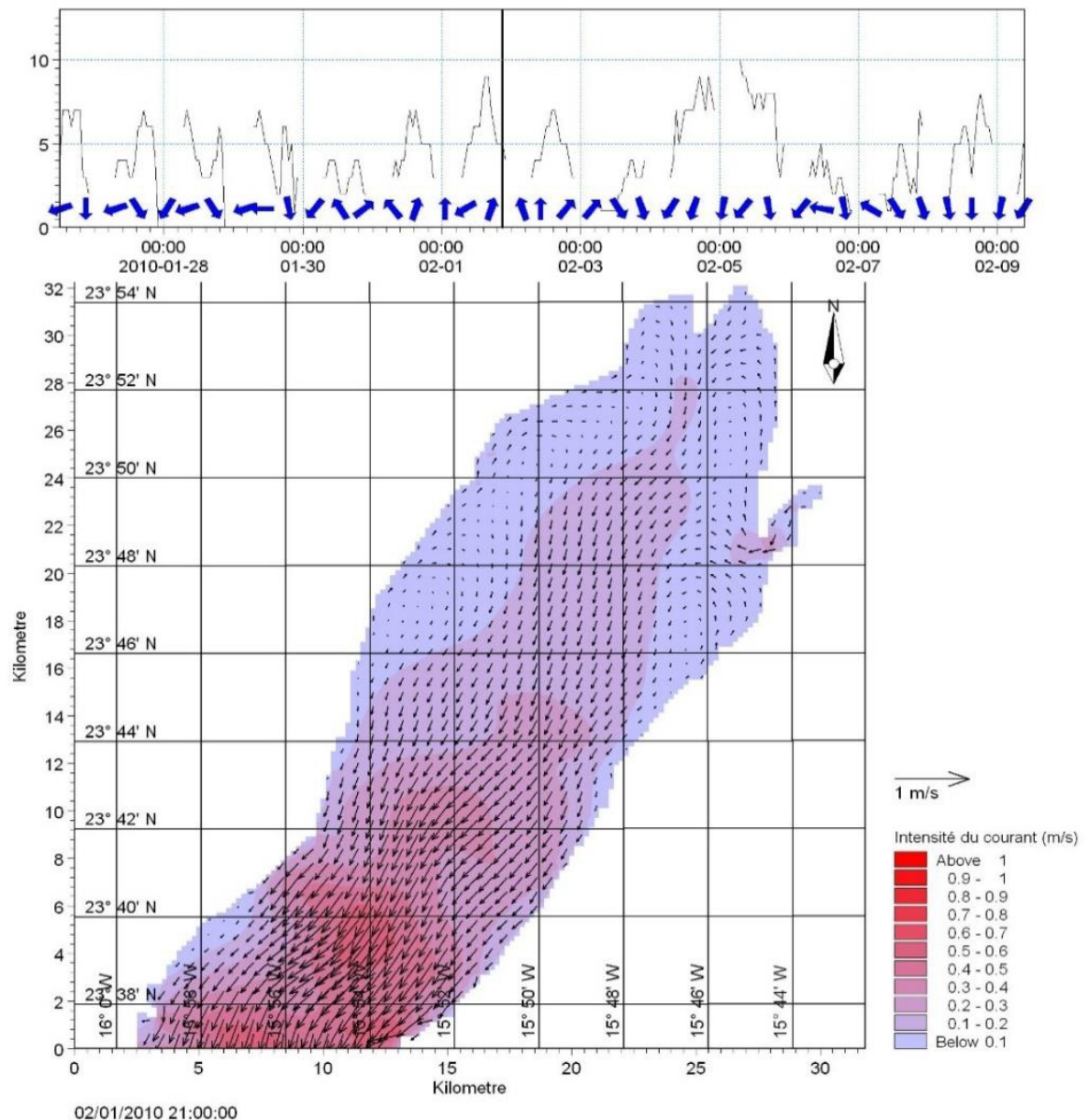

Figure 9. Exemple de circulation de la baie de Dakhla en période de vive-eau à marée sortante (cas du 01 février 2010 à 21h-coefficient de marée 107). 


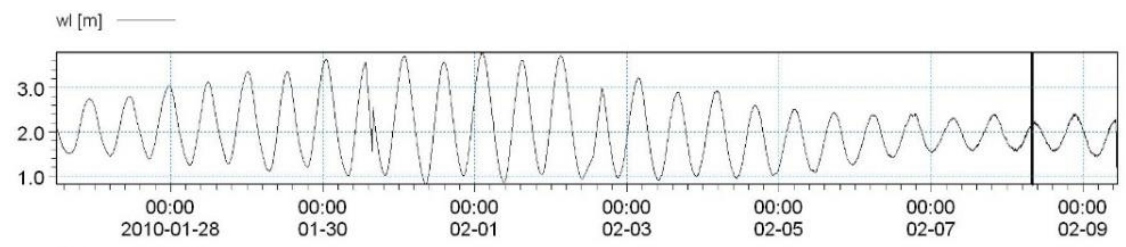

wind speed $[\mathrm{m} / \mathrm{s}]$
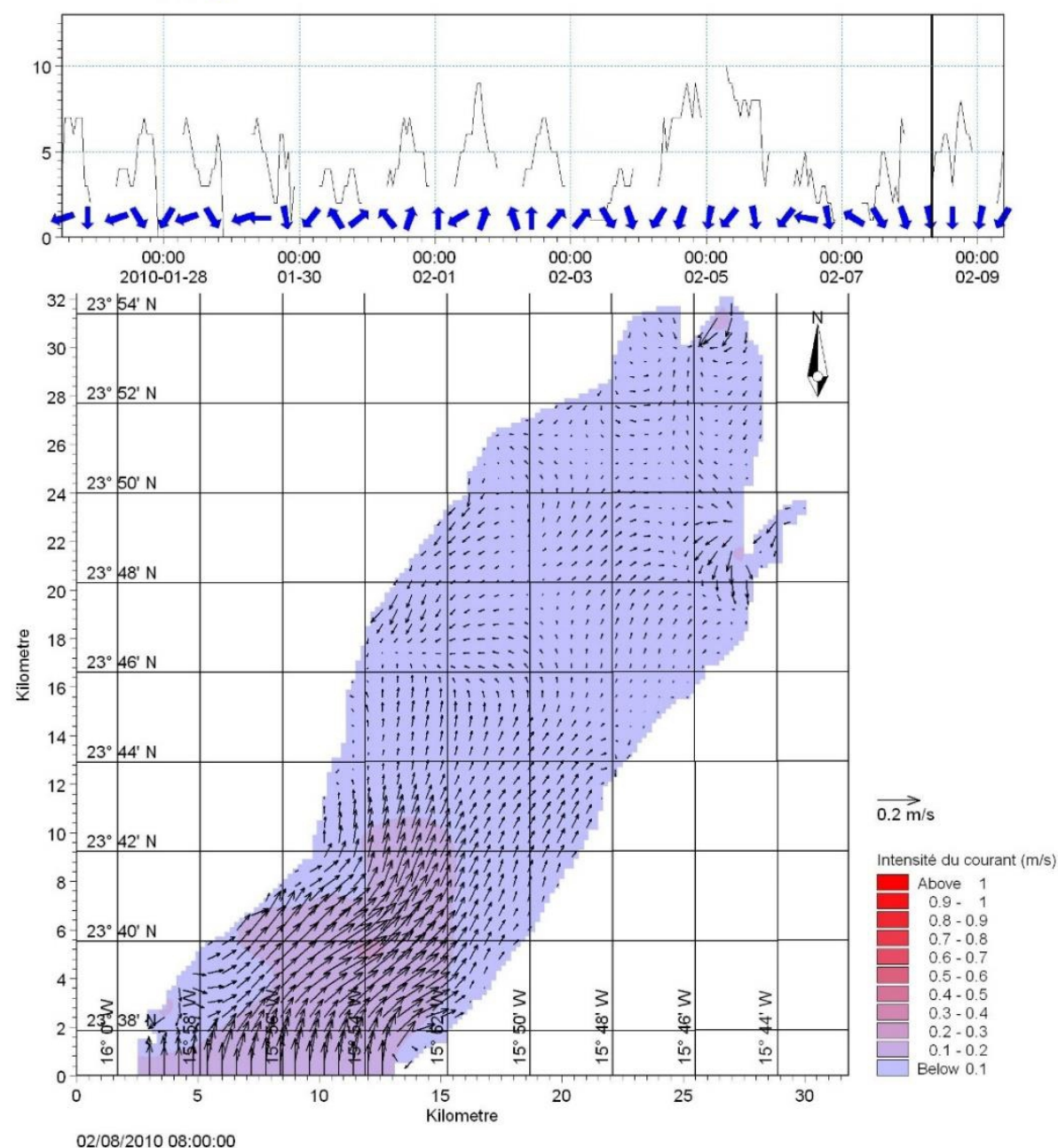

Figure 10. Exemple de circulation de la baie de Dakhla en période de morte-eau à marée entrante (cas du 08 février 2010 à $8 \mathrm{~h}$ - coefficient de marée 20). 


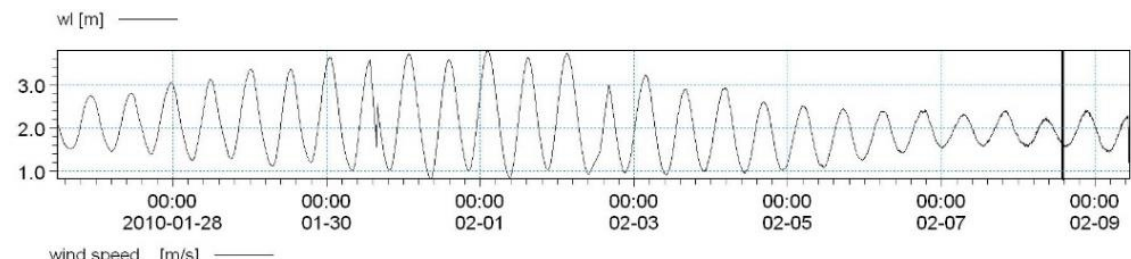

wind speed $[\mathrm{m} / \mathrm{s}]$
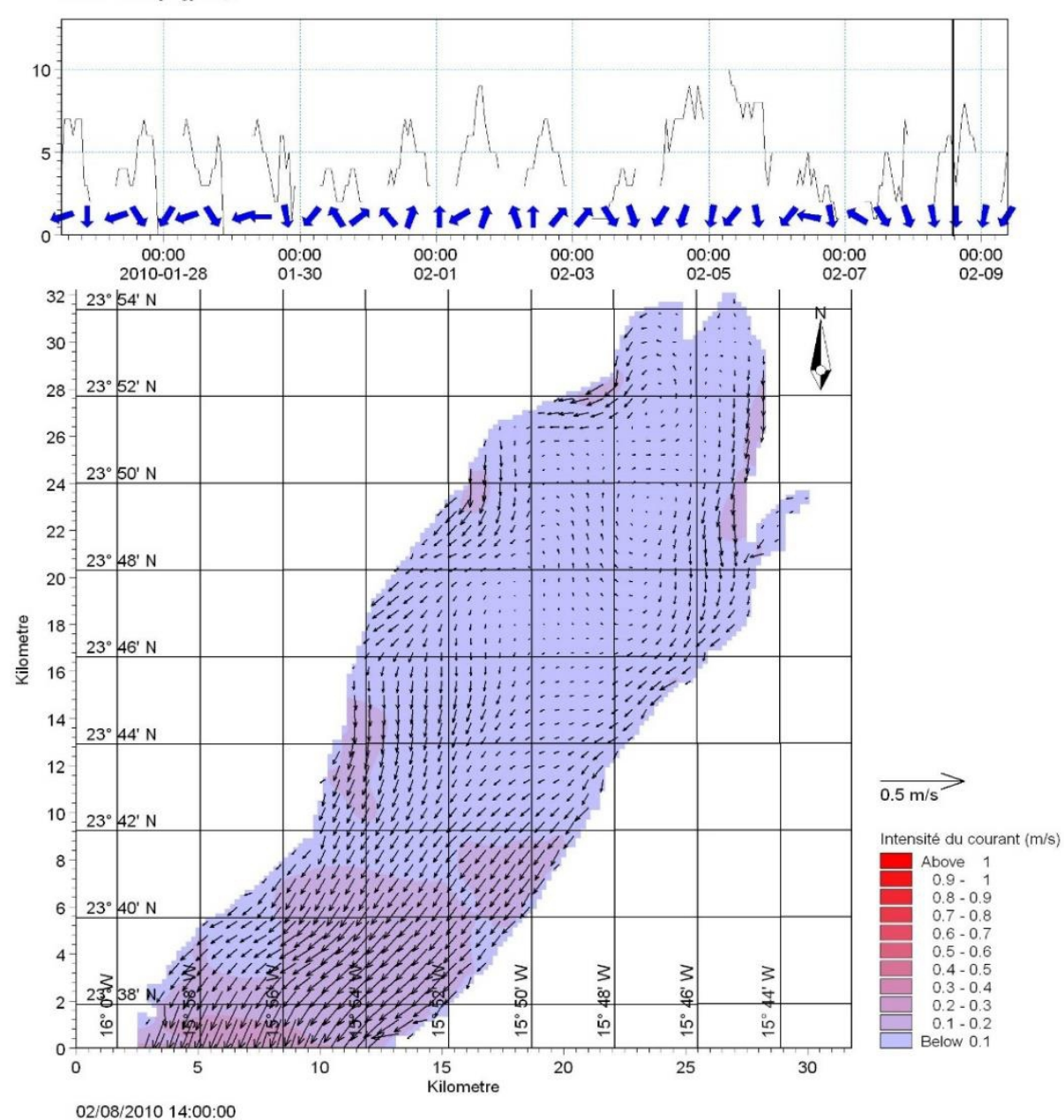

Figure 11. Exemple de circulation de la baie de Dakhla en période de morte-eau à marée sortante (cas du 08 février 2010 à $14 \mathrm{~h}$ - coefficient de marée 23).

Quelque soit le type de marées de vive-eau et de morte-eau, les courants de marée sont bidirectionnels dans l'axe Nord Est - Sud Ouest de la baie en suivant les cycles de la marée semi diurne (M2). En périodes de marées de vives-eaux (coefficients de marée entre 103 et 107) et durant les 
flots, on constate que les plus forts courants se manifestent généralement en aval de la baie entre les latitudes $23^{\circ} 38^{\prime} \mathrm{N}$ et $23^{\circ} 44^{\prime} \mathrm{N}$ ( soit entre Lasarga et Dakhla sur la rive Ouest de la baie) (figures 8 et 9). Sur la rive Est de la baie, les plus forts courants dont les intensités sont comprises entre 1 et $2 \mathrm{~m} \cdot \mathrm{s}^{-1}$ se manifestent principalement entre Hoja Lalmera, El Argoub et Puertito. Vers l'amont de la baie et au delà des latitudes supérieures à $23^{\circ} 44^{\prime} \mathrm{N}$ (soit entre Dakhla, Boutalha et en allant vers les parcs aquacoles), les intensités du courant sont faibles, comprises généralement entre 0.1 et $0.4 \mathrm{~m} . \mathrm{s}^{-1}$ (figures 8 et 9). Durant les jusants et dans la zone centrale et vers l'amont de la baie, des cellules gyratoires cycloniques vers la rive Ouest et/ou anticycloniques vers la rive Est du courant sont observées, suite à une bathymétrie non régulière et peu profonde et la présence d'une multitude de chenaux dans ces zones (Figure 5). En périodes de marées de mortes-eaux (coefficients de marée entre 20 et 23), les courants de marée sont ainsi plus faibles dont les intensités sont inférieures à $0.4 \mathrm{~m} \cdot \mathrm{s}^{-1}$ dans la baie (figures 10 et 11). On constate que les plus forts courants se manifestent à l'entrée de la baie, entre La Sarga (coté Ouest de la baie) et Puertito-Argoub (côté Est de la baie). Dans la zone centrale et vers l'amont de la baie et durant les jusants, on observe également ces cellules gyratoires du courant. Bien que faibles et dû à l'influence des vents, ces cellules vont générer près des côtes des courants de dérive. Une autre particularité de la circulation marine de la baie est que les courants de marée sortants (jusants) sont généralement légèrement plus intenses que les courants entrants (flots), que ce soit en marées de vives eaux ou de mortes eaux (Figures 8 à 11).

\section{Conclusion}

Cette étude a visé à étudier la circulation marine de la baie de Dakhla et à définir son mode de fonctionnement hydrodynamique par modélisation hydrodynamique 2D sur la période allant du 28 janvier au 09 février 2010. Les résultats de cette étude ont montré que les marées, principalement la marée semi diurne de type M2 qui est dominante sur la façade atlantique marocaine, dominent les courants de marée au sein de la baie. Durant les marées de vive eau, les courants sont très intenses (entre 1 et $2 \mathrm{~m} \cdot \mathrm{s}^{-1}$ ) au niveau de l'entrée de la baie et à son voisinage, inférieures à $1 \mathrm{~m} \cdot \mathrm{s}^{-1}$ dans la partie centrale de la baie et inférieures à $0.5 \mathrm{~m} . \mathrm{s}^{-1}$ en se dirigeant vers l'amont de la baie. Durant les marées de mortes eaux, les intensités du courant de marée sont généralement moindres et inférieures à $0.5 \mathrm{~m} . \mathrm{s}^{-1}$ dans la baie. D'autres travaux par modélisation hydrodynamique 3D de la baie sont en cours de réalisation, en vue d'estimer le temps de renouvellement des eaux de la baie et d'autres facteurs régissant son fonctionnement hydrodynamique. 


\section{Remerciements}

Cet article est dédié à la mémoire de feu Dr J.Lakhdar Idrissi, chercheur à l'Institut National de Recherche Halieutique qui a beaucoup travaillé sur la baie de Dakhla avant son décès en 2010. Les auteurs tiennent aussi à remercier l'équipe du Centre Régional de l'INRH à Dakhla qui a contribué à la mission de terrain ainsi que les évaluateurs externes anonymes de la revue.

\section{References :}

1. ANDA (2015). Appel à Manifestation d'Intérêt - Plan de développement de l'aquaculture marine dans la région de Dakhla Oued Eddahhab.. Agence Nationale pour le Développement de l'Aquaculture, Royaume du Maroc, 24p.

2. Arid, H., Moudni, H.,Orbi, A., Talbaoui, M., \& Idrissi, J.I. (2005). Télédétection spatiale et SIG pour la gestion intégrée des potentialités aquacoles, Geobservateur, ISSN 113-4410, $\mathrm{N}^{\circ} 14$, Novembre 2005, p. 49-62.

3. Bergier, P., Qninba, A. \& M. Thevenot, M. (2013). Notes naturalistes au Sahara Atlantique marocain - 3. Go-South Bull. (2011), 8, 67 103.

4. Danish Hydraulic Institute (1990). Coastal Hydraulics and Oceanography. Mike 21. Hydrodynamic Module. User's Guide and reference Manual.

5. Kamara, A., Rharbi, N., Berraho, Ab., \& Ramdani, M. (2005). Etude comparative du cycle sexuel de la palourde Ruditapes decussatus issue de trois milieux paraliques des côtes marocaines, Mar. Life 2005 - VOL.15 (1-2): 43-50.

6. Kamara, A., Rharbi., N., Ramdani, M. \& Berraho, Ab. (2008). Recherches préliminaires au développement de l'élevage de la palourde européenne (Ruditapes decussatus L.) sur les côtes marocaines et au repeuplement des sites surexploités. Bull. Soc. zool. Fr., 133(1-3): 193-202.

7. INRH (2002). Ressources littorales: Etat de nos connaissances. Institut National de Recherche Halieutique, Document du Ministère des Pêches Maritimes, Royaume du Maroc, $110 \mathrm{p}$.

8. Lakhdar, I.J., Orbi, A. \& Hilmi, K. (2010). Rapport relatif à l'étude bathymétrique de la baie de Dakhla. Rapport interne, Institut National de Recherche Halieutique, Royaume du Maroc, 22p.

9. NOVEC (2012a). Etude de la vulnérabilité de la baie d'Oued Eddahab - Etat des lieux actuels de la baie d'Oued Eddahab, Rapport inédit, $115 \mathrm{p}$. 
10. NOVEC (2012b). Etude de la vulnérabilité de la baie d'Oued Eddahab - Diagnostic des stratégies et des risques, Rapport inédit, $54 \mathrm{p}$.

11. Orbi, A., Dafir, J.M. \& Berraho, Ab. (1995). Étude pluridisciplinaire de la baie de Dakhla. Rapport interne, Institut Scientifique des Pêches Maritimes, Royaume du Maroc, 86, 26 p.

12. Orbi, A., Guelorget O. \& Lefebvre, A. (1996). La baie de Dakhla: Organisation biologique et fonctionnement. Rapport interne, Institut National de Recherche Halieutique, Royaume du Maroc, 240 p.

13. Orbi, A. \& Berraho, Ab. (1999). Potentialités de l'aquaculture marocaine. Aquaculture planning in Mediterranean countries. Zaragoza: CIHEAM, 1999. p. 77-81 - Cahiers Options Méditerranéennes, $\mathrm{N}^{\circ} 43$.

14. Saad, Z., Orbi, A., Abouabdellah, R., Saad, A. \& Oudra, B. (2013). Impact of economic development on the dynamics of phytoplankton and physic-chemical quality of Dakhla Bay (South of Morocco). South Asian Journal of Experimental Biology, 3 (5), 274-285.

15. Saad, Z., El-Gharmali., A., El-Khalloufic, F., Saad., A., Oudra, B. (2015). Metal contamination of the macrobenthic fauna of the Dakhla Bay (South of Morocco): an ecotoxicological diagnostic and environmental perspective. South Asian Journal of Experimental Biology 2015 Vol.5 No.2 pp.55-69.

16. SHOM. Consulter le site web du SHOM (www.shom.fr)

17. Zidane, H., Orbi, A., Mouradi, A., Zidane, F. \& Blais, J-F. (2008). Structure hydrologique et édaphique d'un site ostreicole: Duna Blanca (la baie de Dakhla sud du Maroc). Environnemental technology, Vol. 29, pp 1031-1042.

18. Zidane, H. (2011). Potentialités aquacoles de la baie de Dakhla. Environnemental technology, Vol. 29, pp 1031-1042.

19. Zidane, H., Menioui, M., Mouradi, A., Orbi, A. \& Zidane, F. (2013a). Croissance de l'huître C. Gigas, en élevage dans la baie de Dakhla Maroc, Rapp. Comm. int. Mer Médit., CIESM 40. p. 470.

20. Zidane, H., Menioui, M., Mouradi, A., Orbi, A., Zidane, F. \& Blais, J-F. (2013b). Les métaux lourds dans le sédiment de et leur impact sur le développement de la conchyliculture (Baie de Dakhla, Maroc). Rapp. Comm. int. Mer Médit., CIESM 40, p. 766. 